\title{
PROPAGATION AND BIFURCATION OF CRACKS BASED ON IMPLICIT SURFACES AND KINEMATIC VELOCITIES
}

\author{
V.A. KOVTUNENKO ${ }^{\dagger}$, K. KUNISCH${ }^{\diamond}$, AND W. RING ${ }^{\diamond}$ \\ ABSTRACT. We describe cracks and their propagation with a given \\ kinematic velocity as zero-level sets of a scalar non-negative func- \\ tion satisfying a transport equation. For smooth velocities this \\ description is equivalent to the coordinate transformation of a do- \\ main containing the crack. Based on an implicit surface formula- \\ tion we investigate numerically bifurcation type phenomena of the \\ crack such as branching and merging. Analytical and computa- \\ tional examples of the crack evolution with continuous as well as \\ discontinuous velocities are presented in 2D and 3D domains.
}

\section{INTRODUCTION}

The problem of the appropriate description of finite propagation or infinitesimal perturbation of a geometric variable such as the shape of an object arises in various applications. Optimal shape design and identification of shapes, which are not accessible to direct measurements as e.g. in non-destructive testing or mine detection are concrete examples. Classical techniques are based on perturbations of the identity operator or (equivalently) on the velocity method. These techniques can be used for the description of homeomorphic displacements of geometric objects with regular boundaries and for the calculation of sensitivities of geometry dependent functionals [29], [17], [8].

Geometric methods involving level sets can also describe the movement of a shape variable [22], [27], [20], [4], [13], [21]. Level set methods can be used in a natural way to define shape perturbations and to obtain sensitivity results, as was observed in [5], [12]. The geometric variable is described as the zero level-set of a function $\phi$ (which is often chosen as the signed-distance function to the boundary of the shape

1991 Mathematics Subject Classification. 49J40, 49Q10, 49Q12, 49L99, 73M25.

Key words and phrases. crack propagation, bifurcation, level set, implicit surface, velocity method.

† Lavrent'ev Institute of Hydrodynamics, Novosibirsk, Russia.

$\diamond$ Institute for Mathematics and Scientific Computing, University of Graz, Austria. 
variable) and propagated by solving an equation of Hamilton-Jacobi type:

$$
\phi_{t}+V_{n}|\nabla \phi|=0
$$

where $V_{n}$ is scalar velocity. It is known that under appropriate condition on $V_{n}$, the Hamilton-Jacobi equation (the "level-set equation") has a unique viscosity solution which exists for all times [19], [7] in contrast to classical methods which are inherently local. The major advantage of the level set method, however, lies in its flexibility, which allows to treat topology changes for the geometric variable in a unified way, and in its relatively simple implementation on fixed rectangular grids.

In this work, the geometric objects in which we are especially interested are cracks, i.e. subsets of $\mathbb{R}^{N}$ with Hausdorff dimension $N-1$. Crack perturbation techniques are necessary in the framework of fracture mechanics for the determination of the direction and speed of propagation of cracks in solids [11], [31], [30]. It is generally accepted that a local fracture criterion involves the energy release rate at the crack tip, which can be expressed as a shape derivative of a potential energy functional [26]. Using shape sensitivity analysis for models in non-smooth domains with rectilinear cracks subject to inequality constraints, representations of the shape derivative were obtained in [15], [16], [18]. These considerations were based on perturbations of the identity operator and the corresponding coordinate transformations of domains with cracks.

In this paper the description of cracks and their propagation motivates our interest in non-parameterized objects of Hausdorff dimension one in two-dimensional space and Hausdorff dimension two in three-dimensional spaces. This specifically includes nonclosed, possibly branching curves in $\mathbb{R}^{2}$ or surfaces with nonempty boundaries in $\mathbb{R}^{3}$. In the case of cracks in three dimensions these boundaries correspond to crack fronts. We aim for describing the evolution of cracks including phenomena such as bifurcation of tips in $\mathbb{R}^{2}$ and bifurcation of crack fronts in $\mathbb{R}^{3}$. This goal motivates our choice of representing the crack by an implicit surface (the zero-level set of a non-negative time-dependent function $\rho$ ).

Level set methods are used in the classical context for the evolution of a dynamic surface with the velocity having non-zero component normal to the surface. Opposite to this case, the presented examples resulting a crack propagation phenomena have zero normal component of the velocity, and only the tangential component is non-zero. The former case is realized with the help of the level-set equation $(H-J)$, and the latter case is described by a transport equation $(T)$ following later. 
This inherent specialty distinguish the proposed method of an implicit surface description with the other (classical) level set methods.

Before we enter into the details let us recall the seminal work of Osher and collaborators [3] in which vector-valued level set functions were proposed as a technique to describe the evolution of surfaces with codimension higher than one. This is a very powerful approach for the description of the evolution of surfaces without boundaries with the goal of preserving merging and breaking properties. In [33] vector valued level set functions were proposed for the description of the evolution of cracks. This approach was combined with a maximumhoop-stress criterion to determine the direction of the movement of the crack tip. However, it is not primarily geared towards bifurcation type phenomena.

Vector-valued level set functions were studied earlier in [1] and [2] with the goal of analyzing well-posedness. From the analytical point of view, the vector valued approach is difficult to treat because it leads to a system of partial differential equations for which no maximum principle is available. This would be necessary for the viscosity sub- and super-solution concepts to apply. Therefore, the authors in [1,2] introduced a single non-negative level set function for the representation of the propagating surface. Here we utilize the same approach but focus on its numerical realization. In particular we shall address potential difficulties related to the accurate determination of the location of the zero level set. Our numerical strategy involves a sharpening procedure for its correct determination without "thickening" phenomena observed in $[3]$.

If the crack is defined by an implicit surface which is expressed as the zero level set of a non-negative function $\rho$ propagated by a given vector field $V$, and all data are sufficiently smooth, then $\rho(t, y)$ satisfies

$$
\rho_{t}+V \nabla \rho=0
$$

It is easily seen that $(H-J)$ is the specific case of $(T)$ where $V=V_{n} n$ with a scalar velocity $V_{n}$ and $n$ the direction normal to the crack.

We shall utilize the implicit formulation $(T)$ in two different ways. Suppose first, that we are given a moving family of cracks $\Gamma_{t}, t \geq 0$. It is quite natural to define $\rho^{d}(t, y)=\operatorname{dist}\left(y, \Gamma_{t}\right)$ as the corresponding level set function for which $\Gamma_{t}=\left\{y: \rho^{d}(t, y)=0\right\}$. Solving the algebraic equation

$$
\rho_{t}^{d}+V \nabla \rho^{d}=0
$$


for $V$, a natural extension of the velocity vector field (which a-priori is defined only on $\Gamma$ ) onto a neighborhood of the moving crack is obtained. Such an extension is a necessary ingredient for any kind of shape sensitivity analysis in the classical and in the level set context. Construction of extension velocities $(A)$ for certain families of moving cracks are given, and the equivalence between the implicit formulation $(T)$ and the propagation of the crack via coordinate transformation methods are proved in Section 2. The corresponding transformation function and its inverse are constructed as the solutions of non-linear ODEs and transport equations, respectively, where the method of characteristics is used to establish the connection.

The main objective of the paper is the numerical investigation of moving cracks in the framework of implicit surfaces, especially the capability of the method to describe non-parameterizable cracks. In Section 3 numerical calculations of the propagation of cracks with given velocities are presented in 2D and 3D domains including bifurcating cracks. We compare two algorithms, where one is based on solution of ODEs and the other is based on the transport equation $(T)$. The algorithms are realized by high-accurate Runge-Kutta and WENO schemes respectively [23], [14].

\section{CRACKS AND Their PROPAGation BASED ON IMPlicit SURFACES}

In this section we discuss the use of scalar non-negative functions to describe the movement of cracks with a given velocity. We also relate the implicit surface description to the classical formulation of crack movement using coordinate-transformations based on the velocity vector field. Clearly the implicit approach is more general since it is more readily applicable to discontinuous velocity vector fields and hence the cracks for different times need not be homeomorphic. Thus the implicit formulation allows topology changes such as branching and merging of crack branches.

Consider at time $t=0$ the crack $\Upsilon_{0}$ as a compact subset of $\mathbb{R}^{N}$, $N=2$ or 3 with Hausdorff dimension $(N-1)$ and choose a continuous function $\rho_{0} \geq 0$ such that

(1) $\Upsilon_{0}=\left\{x \in \mathbb{R}^{N}: \rho_{0}(x)=0\right\}, \quad \mathbb{R}^{N} \backslash \bar{\Upsilon}_{0}=\left\{x \in \mathbb{R}^{N}: \rho_{0}(x)>0\right\}$.

The distance function $\rho_{0}(x)=\inf _{y \in \Upsilon_{0}}|x-y|$, for instance, which is Lipschitz continuous in $\mathbb{R}^{N}$ (see $[8$, Thm.2.1, p.154]) serves this purpose. To describe the movement of the crack we choose a velocity field 
$V=V(t, x)$ and consider the transport equation (see [9]):

$$
\left\{\begin{array}{l}
\frac{\partial \rho}{\partial t}(t, y)+V(t, y) \nabla \rho(t, y)=0 \\
\rho(0, y)=\rho_{0}(y)
\end{array}\right.
$$

Under appropriate conditions the solution to (2) satisfies $\rho(t) \geq 0$ and we can define the crack at time $t$ by the implicit surface

$$
\Upsilon_{t}=\left\{y \in \mathbb{R}^{N}: \rho(t, y)=0\right\}
$$

It will be convenient to first recall the situation in the case of smooth vector fields. Below the subscript $u$ denotes global uniform Lipschitz continuity of the elements in the respective function space.

Lemma 2.1. Assume that $V=\left(V_{1}, \ldots, V_{N}\right) \in C\left([0, \infty) ; C_{u}^{0,1}\left(\mathbb{R}^{N}\right)\right)^{N}$ and $\rho_{0} \in C_{u}^{0,1}\left(\mathbb{R}^{N}\right)$ with $\rho_{0} \geq 0$. For arbitrary $T>0$ the Cauchy problem (2) has a unique solution $\rho \in C_{u}^{0,1}\left((0, T) \times \mathbb{R}^{N}\right)$ which satisfies (2) point-wise almost everywhere. Moreover, the solution has the form

$$
\rho(t, y)=\rho_{0}\left(R^{-1}(t, y)\right)
$$

with an invertible mapping $R \in C^{1}\left([0, T] ; C_{u}^{0,1}\left(\mathbb{R}^{N}\right)\right)^{N}$ such that $R^{-1} \in$ $C_{u}^{0,1}\left((0, T) \times \mathbb{R}^{N}\right)^{N}$. If $\rho_{0} \geq 0$ we also have $\rho \geq 0$.

The proof is standard and can be based on the characteristic equations

$$
\left\{\begin{array}{l}
\frac{d R}{d t}(t)=V(t, R(t)) \\
R(0)=x
\end{array}\right.
$$

Due to the regularity assumption on $V$ and classical results on existence to dynamical systems, see e.g. [8, Thm.4.1], it follows that (5) admits a unique solution $R(t, \cdot)$ satisfying

$$
R \in C^{1}\left([0, T] ; C_{u}^{0,1}\left(\mathbb{R}^{N}\right)\right)^{N} \text { and } R^{-1} \in C\left([0, T] ; C_{u}^{0,1}\left(\mathbb{R}^{N}\right)\right)^{N} .
$$

It can be shown that

$$
\rho(t, y)=\rho_{0}\left(R^{-1}(t, y)\right)
$$

satisfies (2) almost everywhere on $(0, T) \times \mathbb{R}^{N}$. Moreover $S(t, \cdot)=$ $R^{-1}(t, \cdot)$, where the components of $S$ are solutions to the system of transport equations

$$
\begin{cases}\frac{\partial S_{i}}{\partial t}(t, y)+V(t, y) \nabla S_{i}(t, y)=0 & , i=1, \ldots, N \\ S_{i}(0, y)=y_{i} & , \quad i=1, \ldots, N\end{cases}
$$

which follows that $R^{-1} \in C_{u}^{0,1}\left((0, T) \times \mathbb{R}^{N}\right)^{N}$. By differentiating with respect to $y$ the relation

$$
y=R\left(t, R^{-1}(t, y)\right), \quad t \in(0, T),
$$


the inverse function $R^{-1}$ admits an increase of smoothness in $t$ which must be compensated with a decrease of the corresponding smoothness in $y$ :

$$
R^{-1} \in C^{1}\left([0, T] ; L^{\infty}\left(\mathbb{R}^{N}\right)\right)^{N}
$$

Thus given a velocity field $V$, the solution to (2) describes the crack by means of (3) at time $t$ and $y=R(t, x)$ provides the coordinate transformation between $\Upsilon_{0}$ and $\Upsilon_{t}$.

Conversely, given a coordinate transformation $R$ between cracks evolving in time, satisfying (6) and $R(0, x)=x$, one can determine a velocity field to find a function $\rho$ expressing these cracks as zero-level sets. Indeed, such a velocity $V$ is given by

$$
V(t, y)=\frac{\partial R}{\partial t}\left(t, R^{-1}(t, y)\right)
$$

The function $R$ is then a solution to problem (5), and $R^{-1}$ to (8). When $\rho_{0} \geq 0$ describes the crack $\Upsilon_{0}$ as in (1), the function $\rho(t) \geq 0$ obtained from (7) describes the crack $\Upsilon_{t}$ by (3).

Concerning the propagation of cracks, the restriction of $V(t)$ from $\mathbb{R}^{N}$ onto the crack $\Upsilon_{t}$ determines its local direction of propagation, which can be decomposed into normal and tangential components. The former implies changes of the crack shape, and the latter describes its prolongation. Typically the analysis of cracks in fracture mechanics is concerned with the propagation of cracks preserving the previous shapes, resulting in crack propagation in the tangential direction.

Example: Let us consider as a specific example the family of nonplanar cracks defined by

$$
\Upsilon_{t}=\left\{y \in Q: \quad y_{1} \leq \phi\left(t, y_{3}\right), y_{2}=\psi\left(y_{1}, y_{3}\right), 0 \leq y_{3} \leq Y\right\}, \quad t \geq 0,
$$

with $\phi \in C^{1}\left([0, \infty) ; C_{u}^{0,1}(\mathbb{R})\right)$ and $\psi, \frac{\partial \psi}{\partial y_{1}} \in C_{u}^{0,1}\left(\mathbb{R}^{2}\right)$, and $Q=\mathbb{R}^{2} \times$ $(0, Y)$. The cracks can equivalently be expressed as

$$
\Upsilon_{t}=\left\{y \in \mathbb{R}^{3}: \quad \rho(t, y)=0\right\} \cap Q
$$

with the functions of anisotropic distance

$$
\rho(t, y)=\left[y_{1}-\phi\left(t, y_{3}\right)\right]^{+}+\left|y_{2}-\psi\left(y_{1}, y_{3}\right)\right| .
$$

To fulfill (2) the velocity field $V$ has to satisfy

$$
\begin{gathered}
-\chi\left(y_{1}-\phi\right) \frac{\partial \phi}{\partial t}+\left(\chi\left(y_{1}-\phi\right)-\operatorname{sign}\left(y_{2}-\psi\right) \frac{\partial \psi}{\partial y_{1}}\right) V_{1} \\
+\operatorname{sign}\left(y_{2}-\psi\right) V_{2}-\left(\chi\left(y_{1}-\phi\right) \frac{\partial \phi}{\partial y_{3}}+\operatorname{sign}\left(y_{2}-\psi\right) \frac{\partial \psi}{\partial y_{3}}\right) V_{3}=0
\end{gathered}
$$


almost everywhere in $(0, T) \times \mathbb{R}^{3}$, where $\chi$ is the characteristic function of the set $\{x>0\}$. The velocity chosen as

$$
V(y)=\left(\frac{\partial \phi}{\partial t}, \frac{\partial \phi}{\partial t} \frac{\partial \psi}{\partial y_{1}}, 0\right)
$$

fulfills the above relation and provides the one-to-one correspondence with

$$
\begin{aligned}
R(t, x) & =\left(\begin{array}{l}
x_{1}+\phi\left(t, x_{3}\right)-\phi\left(0, x_{3}\right) \\
x_{2}+\psi\left(x_{1}+\phi\left(t, x_{3}\right)-\phi\left(0, x_{3}\right), x_{3}\right)-\psi\left(x_{1}, x_{3}\right) \\
x_{3}
\end{array}\right), \\
R^{-1}(t, y) & =\left(\begin{array}{l}
y_{1}-\phi\left(t, y_{3}\right)+\phi\left(0, y_{3}\right) \\
y_{2}-\psi\left(y_{1}, y_{3}\right)+\psi\left(y_{1}-\phi\left(t, y_{3}\right)+\phi\left(0, y_{3}\right), y_{3}\right) \\
y_{3}
\end{array}\right) .
\end{aligned}
$$

We note that the regularity requirement of Lemma 2.1 admits singularities like finite corners at the crack front. Consider, for example, the velocity $V=\left(V_{1}, 0,0\right)$ with $V_{1}(y)=\left[y_{3}-c\right]^{+}$, for some $c \in(0, Y)$. Then $V \in C_{u}^{0,1}\left(\mathbb{R}^{3}\right)^{3}$ and the cracks

$$
\Upsilon_{t}=\left\{y \in Q: \quad y_{1} \leq \phi\left(0, y_{3}\right)+t\left[y_{3}-c\right]^{+}, y_{2}=0,0 \leq y_{3} \leq Y\right\}
$$

exhibit a corner at $y_{3}=c$ on the crack front for every $t>0$.

The Lipschitz regularity assumption of Lemma 2.1 for $V$ does not allow the splitting or merging of cracks. This corresponds to the lack of unique solvability of the characteristic equation (5). However, if the Filippov condition

(11) $(x-y) \cdot(V(t, x)-V(t, y)) \geq-K(t)|x-y|^{2}$, with $K \in L^{1}(0, T)$

holds, then solutions to (2) and (5) in the sense of Filippov are welldefined and enjoy the backwards uniqueness property, [6, 10]. The vector-field $V\left(x_{1}, x_{2}\right)=\left(1, \operatorname{sign}\left(x_{2}\right)\right)$, for example, is not continuous but it satisfies (11). Condition (11) was generalized in [24], for instance. These results imply that $(7)$ is still well-defined and that $\rho(t, y) \geq 0$ if $\rho_{0} \geq 0$. For measure theoretic solution concepts, still based on the method of characteristics and preserving nonnegativity in the sense that $\rho_{0} \geq 0$ implies $\rho(t) \geq 0$ we refer to [25].

\section{Numerical CALCUlations of the CRACK PROPAGATiON}

In this part we apply the theoretical results of Section 2 to calculate numerically the propagation of cracks with a given velocity. Based on the equivalent descriptions of moving cracks either by implicit surfaces (IS) or by coordinate transformations (CT) we suggest two corresponding algorithms and compare them for several examples. The examples 
are constructed using tangential velocities to simulate tangential crack growth. A spiral-shaped crack, branching and merging cracks in $\mathbb{R}^{2}$, and a helicoid-shaped crack in $\mathbb{R}^{3}$ are presented.

The propagation of cracks in the continuous case was described in Section 2. Here we give a description of the corresponding finitedimensional approximation and start with the choice of the discretization. Let $\Omega_{h}$ be a grid with mesh-size $h>0$ in the bounded domain $\Omega$, which for simplicity we assume to be time-independent. The continuous time-interval $[0, T]$ is discretized using a step-length $\Delta t>0$ which is chosen in accordance with the CFL-condition for the transport equation $(T)$. The (unknown) crack $\Gamma_{t}$ has co-dimension 1 in $\Omega$ and generally does not coincide with nodal points of $\Omega_{h}$.

We formulate the following discrete problem of finding approximations $\Gamma_{t}^{s}$ of the cracks $\Gamma_{t}$ :

$(P)$ For the given discrete velocity

$$
V^{h}:\{k \Delta t: 0 \leq k \leq T / \Delta t\} \times \Omega_{h} \rightarrow \mathbb{R}^{N},
$$

starting from the initial crack $\Gamma_{0}^{s}$ at $t=0$, located inside $\Omega$, find the crack $\Gamma_{t_{k}}^{s}$ at all time-steps $t \in(0, T)$.

Here the initial crack $\Gamma_{0}^{s}$ is represented either by a finite number of known points $x \in \mathbb{R}^{N}$ or as the zero level-set of an initial function $\rho_{0}$ for which the values on the grid $\Omega_{h}$ are supposed to be known.

3.1. The CT and IS based algorithms. Following the arguments on coordinate transformations in Section 2, we propose to solve $(P)$ based on the first-order non-linear ODE system (5).

\section{Algorithm CT}

(0) Set $k=0$. Initialize the crack $\Gamma_{t_{0}}^{s}=\Gamma_{0}^{s}$ represented by the points $\left\{x_{0}^{1}, \ldots, x_{0}^{l}\right\}$.

(1) Set $t_{k}=k \Delta t$. If $t_{k}<T$ then go to step 2, else STOP.

(2) Find the velocity $V^{h}\left(t_{k}, x_{k}^{j}\right)$ for all $x_{k}^{j} \in \Gamma_{t_{k}}^{s}, j=1, \ldots, l$ by interpolation from nearest grid points in $\Omega_{h}$.

(3) Compute

$$
x_{k+1}^{j}=x_{k}^{j}+\int_{t_{k}}^{t_{k}+\Delta t} V^{h}\left(t_{k}, x(\tau)\right) d \tau \quad \text { for all } x_{k}^{j} \in \Gamma_{t_{k}}^{s}
$$

using a Runge-Kutta method.

(4) Find the crack $\Gamma_{t_{k+1}}^{s}=\left\{x_{k+1}^{j}\right.$ from (12) $\}$.

(5) Update $k=k+1$, go to step 1.

Velocities tangential to $\Gamma_{t}$ preserve the previous crack shape. In this case, we update only the boundary points of the propagating crack in 
step (3) and we add the new boundary point to the set of existing crack points. This procedure was used in the numerical examples below.

In step 2 we used a linear interpolation of the velocity at $x \in \Gamma_{t_{k}}^{s}$ from nearest nodal points of the grid $\Omega_{h}$. For the numerical integration of (12) we suggest a third-order Runge-Kutta method of the form:

$$
\begin{aligned}
& y^{(i)}=\left(1-\alpha_{i}\right) y^{(0)}+\alpha_{i}\left(y^{(i-1)}+\Delta t V^{h}\left(t_{k}, y^{(i-1)}\right)\right) \quad(i=1,2,3), \\
& x_{k+1}^{j}=y^{(3)}, \quad y^{(0)}=x_{k}^{j}, \quad \alpha_{1}=1, \alpha_{2}=\frac{1}{4}, \alpha_{3}=\frac{2}{3} .
\end{aligned}
$$

This makes it necessary to interpolate the velocity also at the auxiliary points $y^{(i-1)}$ in (13).

Second, we utilize the implicit description of cracks (1), (3) to solve $(P)$ and formulate the following algorithm based on the solution of the transport equation (2). Computationally it is difficult to determine accurately the zero-level of the fixed-sign function $\rho$ in (3). Hence, below we introduce the concept of shadow of a crack, which consists of the nodal points within an $\varepsilon$-neighborhood of the crack.

\section{Algorithm IS}

(0) Set $k=0$, start with the non-negative distance function: $\rho^{h}\left(t_{k}, x\right)=\operatorname{dist}\left(x, \Gamma_{0}^{s}\right)$ for all $x \in \Omega_{h}$.

(1) Set $t_{k}=k \Delta t$. If $t_{k}<T$ then go to step 2, else STOP.

(2) Define the discrete gradient $\nabla^{h} \rho^{h}\left(t_{k}, x\right)$ for all $x \in \Omega_{h}$.

(3) Set $t_{k+1}=t_{k}+\Delta t$, compute $\rho^{h}\left(t_{k+1}, x\right)$ :

$$
\begin{array}{r}
\rho^{h}\left(t_{k+1}, x\right)=\rho^{h}\left(t_{k}, x\right)-\int V^{h}\left(t_{k}, x\right) \nabla^{h} \rho^{h}\left(t_{k}, x\right) \Delta t \\
\text { for all } x \in \Omega_{h}
\end{array}
$$

using an appropriate numerical scheme.

(4') Resharpening: Restore the property that $\rho^{\text {sd }}$ is the non-negative distance function of an $(n-1)$-dimensional crack by solution of an eikonal equation.

(4) Find the shadow of the crack:

$$
\Gamma_{t_{k+1}}^{h}(\varepsilon)=\left\{x \in \Omega_{h}: \quad \rho^{s d}\left(t_{k+1}, x\right) \leq \varepsilon\right\} .
$$

(5) Update $t_{k}=t_{k+1}$, go to step 1.

We now discuss some details of the algorithm. Numerical tests showed that the isotropic distance

$$
\operatorname{dist}\left(x, \Gamma_{0}^{s}\right)=\min _{z \in \Gamma_{0}^{s}}\left(\sum_{j=1}^{N}\left(x_{j}-z_{j}\right)^{2}\right)^{1 / 2}
$$


was preferable for the initialization in step 0 over an anisotropic choice of the norm in $\mathbb{R}^{N}$. The latter choice was convenient in the analytical constructions in Section 2 to obtain reference velocities.

For the discrete gradient $\nabla^{h}$ in step 2 we used a 5th-order accurate WENO-approximation based on 6-point finite difference left- and rightbiased stencils, marked below with "-" and "+", respectively. In each coordinate direction $x_{j}(j=1, \ldots, N)$ these approximations for the uniform grid are given by the following formula with respect to $i$-th nodal point [14]:

$$
\begin{aligned}
\frac{\partial^{ \pm}}{\partial x_{j}} \rho_{i} & =\frac{1}{12}\left(-\Delta^{+} \rho_{i-2}+7 \Delta^{+} \rho_{i-1}+7 \Delta^{+} \rho_{i}-\Delta^{+} \rho_{i+1}\right) \\
& \pm W\left(\Delta^{-} \Delta^{+} \rho_{i \pm 2}, \Delta^{-} \Delta^{+} \rho_{i \pm 1}, \Delta^{-} \Delta^{+} \rho_{i}, \Delta^{-} \Delta^{+} \rho_{i \mp 1}\right)
\end{aligned}
$$

with

$$
\begin{aligned}
& \Delta^{+} \rho_{m}=\frac{1}{h}\left(\rho_{m+1}-\rho_{m}\right), \quad \Delta^{-} \Delta^{+} \rho_{m}=\frac{1}{h}\left(\rho_{m-1}-2 \rho_{m}+\rho_{m+1}\right), \\
& W\left(c_{1}, c_{2}, c_{3}, c_{4}\right)=\frac{1}{3} \omega_{0}\left(c_{1}-2 c_{2}+c_{3}\right)+\frac{1}{12}\left(2 \omega_{2}-1\right)\left(c_{2}-2 c_{3}+c_{4}\right), \\
& \omega_{0}=\alpha_{0}\left(\alpha_{0}+\alpha_{1}+\alpha_{2}\right)^{-1}, \quad \omega_{2}=\alpha_{2}\left(\alpha_{0}+\alpha_{1}+\alpha_{2}\right)^{-1}, \\
& \alpha_{0}=\left(\epsilon+i_{0}\right)^{-2}, \quad \alpha_{1}=6\left(\epsilon+i_{1}\right)^{-2}, \quad \alpha_{2}=3\left(\epsilon+i_{2}\right)^{-2}, \\
& i_{0}=13\left(c_{1}-c_{2}\right)^{2}+3\left(c_{1}-3 c_{2}\right)^{2}, \quad i_{1}=13\left(c_{2}-c_{3}\right)^{2}+3\left(c_{2}+c_{3}\right)^{2}, \\
& i_{2}=13\left(c_{3}-c_{4}\right)^{2}+3\left(3 c_{3}-c_{4}\right)^{2}, \quad \epsilon=10^{-6} .
\end{aligned}
$$

Moreover, a finite-difference approximation of the gradient requires an extension $\widetilde{\Omega}^{h}$ of the grid $\Omega_{h}$ outside $\Omega$. For the suggested 7 -point central scheme a linear extension of $\rho$ onto 3 points in each direction outside $\Omega_{h}$ was used.

We realized the finite-difference approximation of (14) by a LaxFriedrichs flux. Various fluxes of upwind and Godunov type were tested. They show comparable performance when the derivative with respect to space variables is approximated by the WENO-scheme. For the Lax-Friedrichs flux (14) takes the form:

$$
\begin{array}{r}
\rho^{h}\left(t_{k+1}, x\right)=\rho^{h}\left(t_{k}, x\right)-\int \sum_{j=1}^{N}\left\{V_{j}^{h}\left(t_{k}, x\right) \frac{1}{2}\left(\frac{\partial^{+}}{\partial x_{j}}+\frac{\partial^{-}}{\partial x_{j}}\right) \rho^{h}\left(t_{k}, x\right)\right. \\
\left.-\max _{x \in \Omega_{h}}\left(V_{j}^{h}\left(t_{k}, x\right)\right) \frac{1}{2}\left(\frac{\partial^{+}}{\partial x_{j}}-\frac{\partial^{-}}{\partial x_{j}}\right) \rho^{h}\left(t_{k}, x\right)\right\} \Delta t
\end{array}
$$

with the "+" and "-" derivatives from (16).

The 3rd-order Runge-Kutta method was used for time integration in (17), too. Following the notation in (13) results in the following scheme 
for (14):

$$
\begin{aligned}
& \rho^{(i)}=\left(1-\alpha_{i}\right) \rho^{(0)}+\alpha_{i}\left(\rho^{(i-1)}-\Delta t \sum_{j=1}^{N}\left\{V_{j}^{h}\left(t_{k}\right) \frac{1}{2}\left(\frac{\partial^{+}}{\partial x_{j}}+\frac{\partial^{-}}{\partial x_{j}}\right)\right.\right. \\
& \left.\left.-\max _{x \in \Omega_{h}}\left(V_{j}^{h}\left(t_{k}\right)\right) \frac{1}{2}\left(\frac{\partial^{+}}{\partial x_{j}}-\frac{\partial^{-}}{\partial x_{j}}\right)\right\} \rho^{(i-1)}\right) \quad(i=1,2,3), \\
& \rho^{h}\left(t_{k+1}\right)=\rho^{(3)}, \quad \rho^{(0)}=\rho^{h}\left(t_{k}\right) .
\end{aligned}
$$

Again, step 2 is repeated by Runge-Kutta iterations (18) to define the discrete gradients $\nabla^{h} \rho^{(i-1)}$ according to (16).

Step 4' is introduced to cope with the problem that the function $\rho$ looses the property of being the distance function of a crack during the iteration of $t_{k}$. This includes the effect that the function $\rho\left(t_{k}\right)$ can become positive at points which should lie on the crack. Thus, detection of the zero level-set becomes an increasingly ill conditioned task as the crack evolves. To overcome this difficulty we suggest the following heuristic procedure:

- Shift the function $\rho$ to obtain $\rho^{d}=\rho-d$ with a constant $d>0$. The new function $\rho^{d}$ has a zero-level set which includes the crack in its interior.

- Calculate the signed distance function $\rho^{\text {sd }}$ to the zero level-set $\Gamma^{d}=\left\{\rho^{d}=0\right\}$ by solving the eikonal equation

$$
\left|\nabla \rho^{s d}\right|=1
$$

with the boundary condition $\rho^{s d}=0$ on $\Gamma^{d}$. Use a fast marching algorithm for the numerical solution [32, 28].

- Shift $\rho^{s d}=\rho^{s d}+d$ to obtained the resharpened function $\rho^{s d}$ as the non-negative distance function.

The heuristic idea behind the procedure described above is that the signed distance function of a "slim" set - the set $\Gamma^{d}$ which includes the crack - contains a set of singularities inside, which is a good approximation of the actual crack. Moreover, if the thickness of the set is approximately constant, the value of the signed distance function along the set of singular points is also approximately constant. Although this set is not resolved explicitly, the crack is much better localized implicitly in the resharpened function than in the original representation.

In step 4 we do not define the crack $\Gamma_{t_{k+1}}$ but only its shadow $\Gamma_{t_{k+1}}^{h}(\varepsilon)$ as a subset of the grid points $\Omega_{h}$. To determine the crack from its shadow an additional algorithmic step must be introduced. In our numerical tests a local piecewise-linear interpolation by a least-squares 
method was successful. Nevertheless, the interpolation procedure required to be handled interactively to obtain an appropriate performance of the crack in each particular example, and, therefore, it was not included in the algorithm.

Note that Algorithm IS is much more involved than Algorithm CT. If Algorithm CT is applicable it is simpler and more effective than Algorithm IS. However Algorithm IS does not require a parameterization of the crack and hence has a broader scope. In the following examples we discuss situations where the use of each of the algorithms has certain advantages.

3.2. Numerical examples. To construct an example for $(P)$ we choose a reference crack $\Gamma_{t}$ and derive an analytical formula for the corresponding velocity $V$ following the lines of Section 2 . Then this velocity and initial crack $\Gamma_{0}$ are taken for the initialization of both algorithms from Section 3.1. Computed cracks are compared to the reference crack.

Let us start with a smooth example of a spiral-shaped crack represented by a parametric curve in $\mathbb{R}^{2}$. It is convenient to use polar coordinates firstly to construct a velocity and then transform it to Cartesian coordinates. In polar coordinates $r, \phi \geq 0$ we define the crack $\Upsilon_{t}$ for $t \in\left[0,1-t_{0}\right)$ with fixed $0<t_{0}<1$ as half-lines:

$$
\Upsilon_{t}=\left\{r=A(1-s), \phi=\kappa(1-s) \text { for } s \in\left(-\infty, t_{0}+t\right)\right\},
$$

for given positive constants $A, \kappa$. We use the implicit representation $\Upsilon_{t}=\{\rho(t, r, \phi)=0\}$ with anisotropic distance:

$$
\rho(t, r, \phi)=\left[A\left(1-t_{0}-t\right)-r\right]^{+}+\left|\phi-\kappa\left(1-t_{0}-t\right)\right| .
$$

The function $\rho$ satisfies the transport equation

$$
\frac{\partial \rho}{\partial t}+V_{r} \frac{\partial \rho}{\partial r}+V_{\phi} \frac{1}{r} \frac{\partial \rho}{\partial \phi}=0 \quad \text { a.e. } \mathbb{R}_{+}^{2}
$$

where the velocity is given in polar coordinates as

$$
\left(V_{r}, V_{\phi}\right)=(-A,-\kappa r) .
$$

Let $\Omega$ be the unit square $\left\{\left(x_{1}, x_{2}\right) \in(0,1)^{2}\right\}$. Now we specify the polar coordinates $(r, \phi)$ around the point $(0.5,0.5)$ in $\Omega$ from the relation:

$$
x_{1}=0.5+r \cos \phi, \quad x_{2}=0.5+r \sin \phi,
$$

and following (20) we define the bounded crack for $t \in(0, T)$ as follows:

$$
\Gamma_{t}=\left\{\begin{array}{l}
x_{1}=0.5+A(1-s) \cos \kappa(1-s), \\
x_{2}=0.5+A(1-s) \sin \kappa(1-s),
\end{array} \text { for } s \in\left(0, t_{0}+t\right)\right\}
$$


with the constants specified as $A=0.5, \kappa=4 \pi, t_{0}=0.1$, and $T=0.9$. Due to the transformation of vectors in polar coordinates:

$$
\left(\begin{array}{l}
V_{1} \\
V_{2}
\end{array}\right)=\left(\begin{array}{rr}
\cos \phi & -\sin \phi \\
\sin \phi & \cos \phi
\end{array}\right)\left(\begin{array}{l}
V_{r} \\
V_{\phi}
\end{array}\right)
$$

the velocity in Cartesian coordinates has the form

$$
\left(\begin{array}{l}
V_{1} \\
V_{2}
\end{array}\right)=\left(\begin{array}{l}
-A r^{-1}\left(x_{1}-0.5\right)+\kappa\left(x_{2}-0.5\right) \\
-A r^{-1}\left(x_{2}-0.5\right)-\kappa\left(x_{1}-0.5\right)
\end{array}\right)
$$

where $r=\left(\left(x_{1}-0.5\right)^{2}+\left(x_{2}-0.5\right)^{2}\right)^{1 / 2}$.

a) velocity $V_{1}$

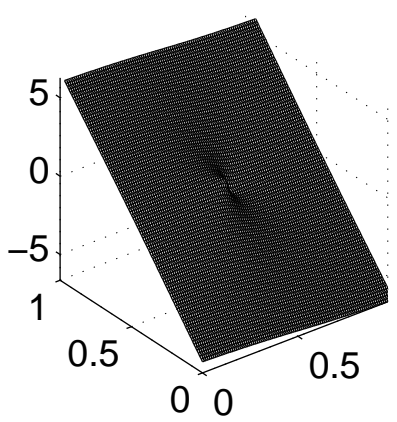

b) velocity $\mathrm{V}_{2}$

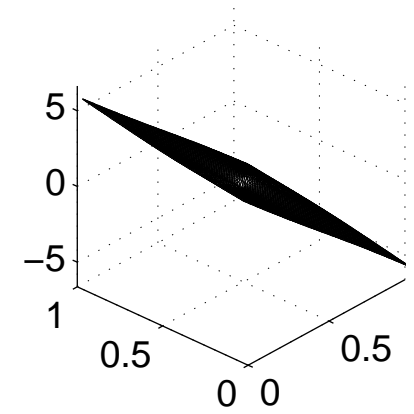

c) $\operatorname{crack} \Gamma_{t}$

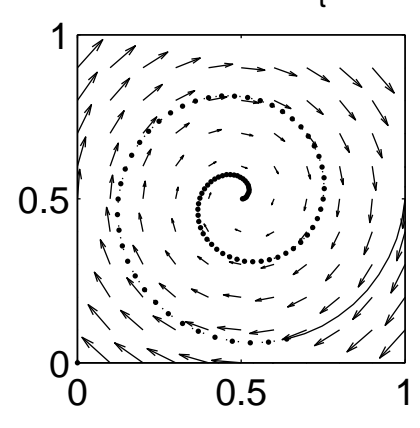

FiguRE 1. The spiral-shaped crack obtained by Algorithm CT.

Next we discretize the data. The domain $\Omega$ is endowed with a uniform grid $\Omega_{h}$. To define $V^{h}$ we restrict $V$ from (23) onto $\Omega_{h}$ and to fix the tip $(1,0.5)$ we set $V^{h}=0$ near the boundary $x_{1}=1$. Both components of this time-independent velocity are presented in Fig. 1 a) and b), respectively. In Fig. 1 c) the velocity field is drawn in $\Omega$, and the initialization $\Gamma_{0}$ obtained from (22) with $t=0$ is depicted with a solid-line.

With these data we solve $(P)$ using Algorithm CT. Choosing $h=$ 0.01 and $\Delta t=0.01$ we calculate a propagation with the velocity $V^{h}$ of the left tip of the initial crack $\Gamma_{0}$ in the time interval $(0,0.9)$. It is depicted by points for 90 time-steps in Fig. $1 \mathrm{c}$ ). For comparison the reference crack $\Gamma_{t}$ from (22) for $t=0.9$ is presented here by a dashed line. For each time-step $t \in(0,0.9)$ it is seen that $\Gamma_{t}^{s}$ is very close to the reference crack $\Gamma_{t}$.

We apply next Algorithm IS with $h=0.01$ and $\Delta t=0.001$ to this example. The numerical results are presented in Fig. 2 a), b) for the initialization, and in Fig. 2 c), d) after 900 time-iterations. The following quantities are depicted: the isotropic distance $\rho_{0}$ to $\Gamma_{0}^{s}$, which 

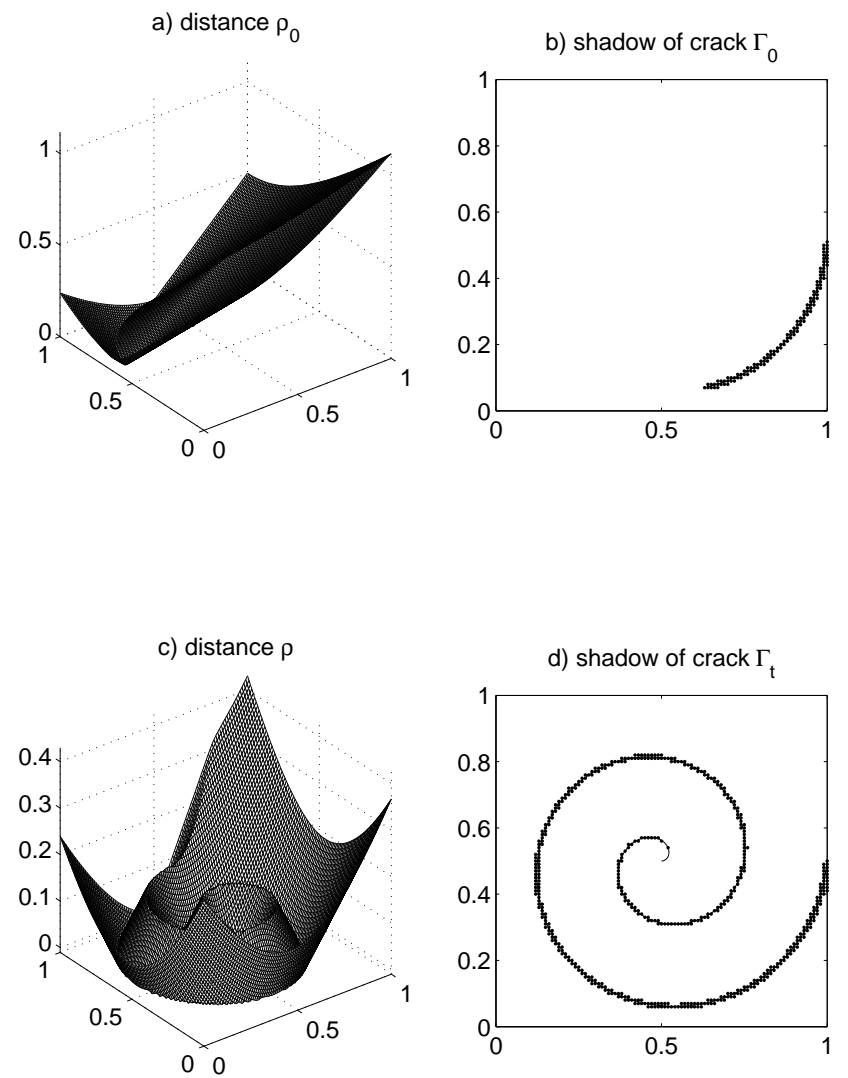

FiguRE 2. The spiral-shaped crack obtained by Algorithm IS.

is discretized with uniform spacing $\Delta s=0.01 \Delta t$ in (22) at $t=0$, the shadow $\Gamma_{0}^{h}(\varepsilon)$ of the initial crack with $\varepsilon=h$, the solution $\rho$ to (18) at $t=0.9$, and the corresponding shadow of the crack with $\varepsilon=h$. The reference cracks are plotted as solid lines in Fig. 2 b) and d), and they are contained within the computed shadows.

In all the examples we used the resharpening strategy with a downshift parameter $d$ which corresponds approximately to two times the mesh-size of the underlying grid. If $d$ is chosen smaller then the fast marching algorithm may produce unstable results.

For this example, the improvement by implementing the resharpening procedure is considerable. Without resharpening the thickness of the shadow region increases constantly during the propagation and does not allow to localize the crack well.

If a velocity vector $V^{\text {tip }}(t)$ is given only at the tip $\Gamma_{t}^{\text {tip }}$ of a propagating crack $\Gamma_{t}$, the question of extending of the velocity to a full velocity field $V(t, x)$ defined on the whole reference domain arises. We suggest 
the following numerical approach which has the property that propagation with the extedend velocity field preserves the shapes obtained at previous time-steps.

As a starting point, we assume that - at every time step $t_{k}$ an (approximate) crack tip $\widetilde{\Gamma}_{t_{k}}^{\text {tip }}$ and a velocity vector $V_{t_{k}}^{\text {tip }}$ has been determined using suitable physical or geometrical arguments. In the level-set context, we usually assume that $\widetilde{\Gamma}_{t_{k}}^{\text {tip }}$ is a regular grid-point.
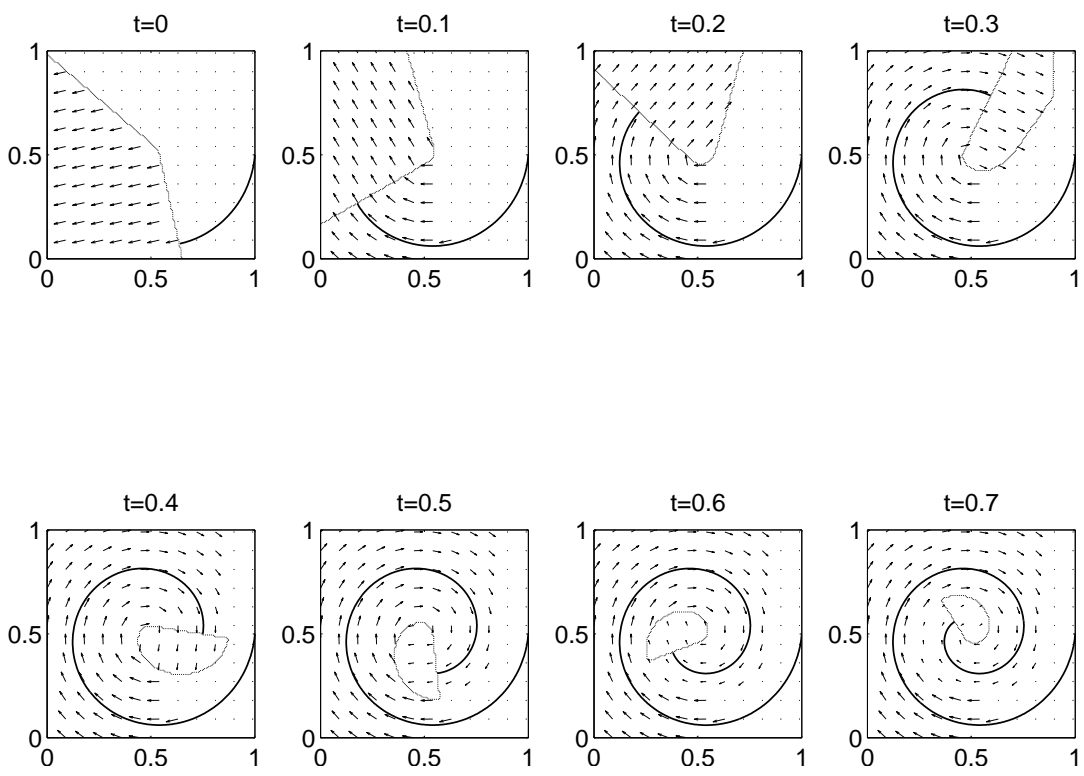

FiguRE 3. Velocity field $V(t)$ extended from the velocity of the crack tip of spiral-shaped crack.

The velocity extension is inserted before step 3 in Algorithm IS:

- Define

$$
\Omega_{u}\left(t_{k}\right)=\left\{x \in \Omega: \quad \operatorname{dist}\left(x, \widetilde{\Gamma}_{t_{k}}^{\mathrm{tip}}\right)=\operatorname{dist}\left(x, \widetilde{\Gamma}_{t_{k}}\right)\right\} ;
$$

- Extend

$$
V\left(t_{k}, x\right)=\left\{\begin{array}{ll}
V_{t_{k}}^{\mathrm{tip}} & \text { if } x \in \Omega_{u}\left(t_{k}\right) \\
V\left(t_{k-1}, x\right) & \text { otherwise }
\end{array} .\right.
$$

The construction of the extension is based on a splitting of the reference domain $\Omega$ into two subdomains. We refer to the first domain $\Omega_{u}\left(t_{k}\right)$ as the update domain where actual movement of the crack takes place. In the update domain the velocity field is set to the constant vector $V_{t_{k}}^{\text {tip }}$. In the complementary domain the velocity is set to the velocity field obtained in the previous time-step. With this choice, the prior shape of the crack is preserved. This is due to the fact that the prior shape 
itself was obtained using the same (tangential) velocity vector field in the complementary domain. As initialization for the first time-step, we use $V\left(t_{-1}, x\right)=0$.

We define the update domain $\Omega_{u}\left(t_{k}\right)$ as the set of all points for which the closest point on the crack is the crack tip. Using this construction, points close to the crack tip in the forward direction propagate with the same velocity as the tip itself. Remote points are not effected by the update velocity and propagate according to the previously specified velocity. In Figure 3 the splitting into two subdomains, the extended velocity and the propagating crack are shown at 8 different time-instances. It can be observed that the update domain is always in front of the crack tip.
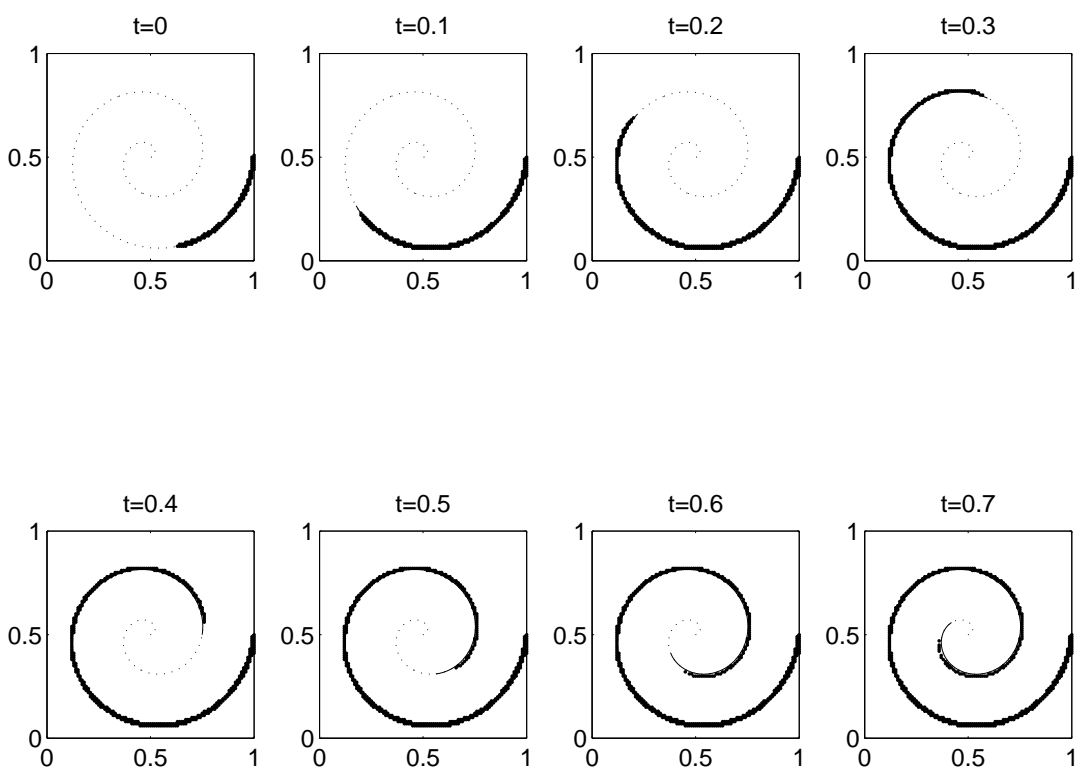

Figure 4. The spiral-shaped crack obtained by Algorithm IS with the velocity extension procedure.

For the numerical calculations shown in Figure 3 we choose $\widetilde{\Gamma}_{t_{k}}^{\text {tip }}$ close to the reference crack tip $\Gamma_{t_{k+1}}^{\mathrm{tip}}$. The latter is found by solving the discrete ODE system (12) at $\Gamma_{t_{k}}^{\mathrm{tip}}$. Nevertheless, $\widetilde{\Gamma}_{t_{k}}^{\mathrm{tip}}$ and $V_{t_{k}}^{\mathrm{tip}}$ can be determined on the fixed grid points of $\Omega^{h}$ without precise tracking of the reference crack. In the example of the spiral-shaped crack we obtain $V^{\text {tip }}(t)$ from the analytical representation (23). The velocity field $V(t, x)$ extended numerically and the sets $\Omega_{u}(t)$ where $\operatorname{dist}\left(x, \widetilde{\Gamma}_{t}^{\text {tip }}\right)=$ $\operatorname{dist}\left(x, \widetilde{\Gamma}_{t}\right)$ are plotted in Fig. 3 at points $t=0,0.1, \ldots, 0.7$. Realization of Algorithm IS with the velocity extension procedure gets the distance 
a) velocity $V_{1}$

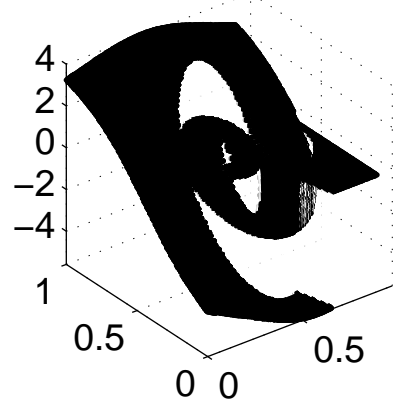

b) velocity $V_{2}$

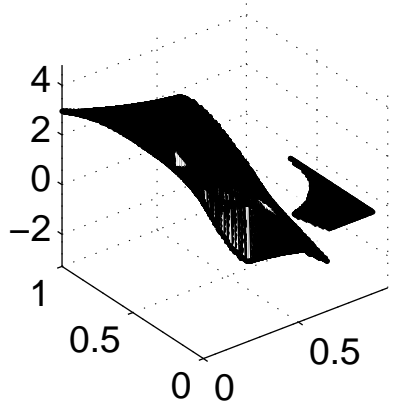

c) velocity field for $\Gamma_{t}$

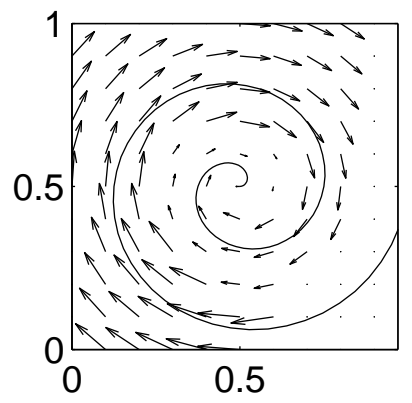

Figure 5. The limit velocity $V$ extended from the velocity of the crack tip of spiral-shaped crack.

function $\rho(t)$ and the respective shadows of crack $\Gamma_{t}$. The latter ones are depicted in Fig. 4 for the selected time points $t$. Note that the limit of iterations of $V\left(t_{k}, x\right)$ in $(24)$ for $k=0,1, \ldots,[0.9 / \Delta t]$ presents a piecewise-constant function, which is depicted in Fig. 5 This timeindependent velocity can be used for Algorithm IS, which in this case produces the same result as plotted in Fig. 4.

From the extension description we get an idea how to construct a piecewise-constant velocity field when the tip of a crack is endowed with a multi-valued vector $V^{\text {tip }}$ at some bifurcation point. The domain of propagation should be splitted into sectors near the bifurcation point such that each value of $V^{\text {tip }}$ could be extended into these sectors with the above procedure. This idea is realized in the next example.
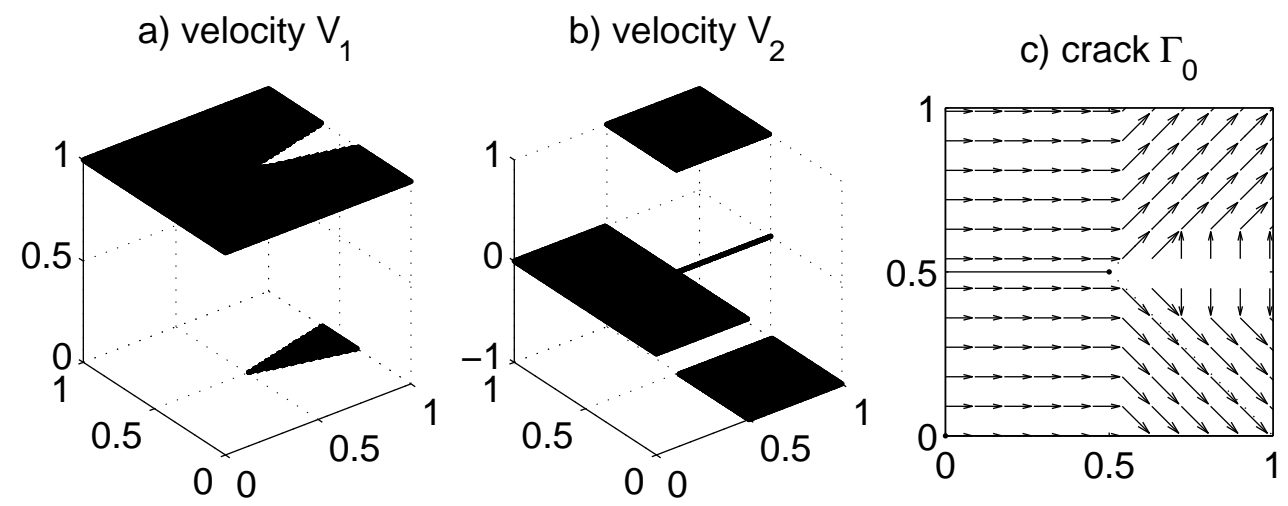

FiguRE 6. Data for the branching crack. 
The second example deals with a non-smooth case of a branching (bifurcating) crack shown in Fig. 6 c) in the unit square $\Omega$ as before. The components $V_{1}$ and $V_{2}$ of the corresponding time-independent velocity are constructed as presented in Fig. 6 a) and b), respectively. The angle of the sector drawn in Fig. 6 a) is chosen as $2 \arctan 1 / 4$. It can be taken arbitrarily within $(0, \pi / 2)$. Note that such a discontinuous velocity is inadmissible in the context of the (classical) consideration in Section 2. In the discrete space we start with the initial rectilinear crack $\Gamma_{0}=\left\{0<x_{1}<0.5, x_{2}=0.5\right\}$ given in Fig. 6 c) with a solidline, which should follow in "time" $t$ two rectilinear branches forming $\pm \pi / 4$-angles with the line $x_{2}=0.5$ marked with dashed-lines.
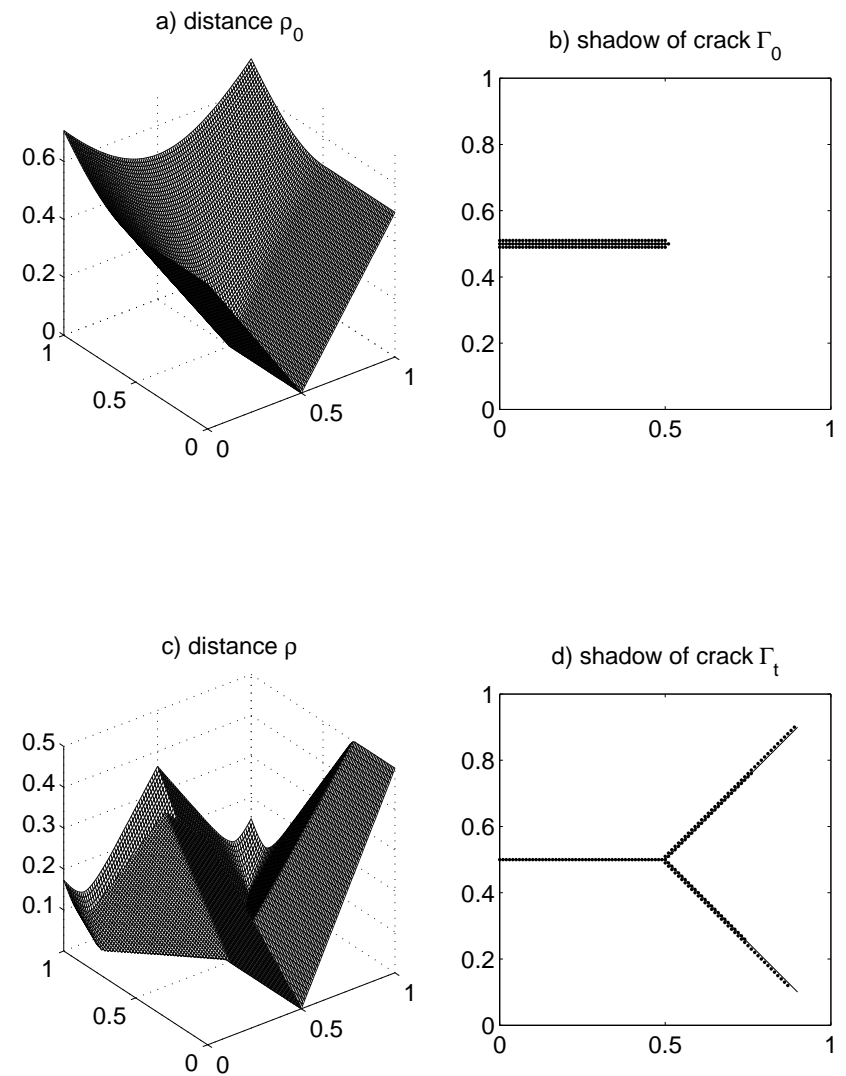

FigURE 7 . The branching crack obtained by Algorithm IS.

Algorithm CT applied to this non-smooth example can follow only one of the branches, thus it is inconsistent. Alternatively, Algorithm IS gives us rather good result as we can see in Fig. 7, where the solution $\rho$ and the corresponding shadow of $\Gamma_{t}$ are computed for $h=0.01$ and $\Delta t=0.001$ after 400 time-steps $(t=0.4)$. The solid-line in Fig. 7 
d) represents the reference crack, whose branches are both closely surrounded by the computed crack shadow with $\varepsilon=h$.

The initialization of $\rho^{h}\left(t_{0}\right)$ should be taken precise enough such that the zero-level sets of $\rho^{h}\left(t_{0}\right)$ and $\rho_{0}$ from (2) were close to each other. Otherwise, the gap of $\rho^{h}\left(t_{k}\right)$ from zero increases constantly during iterations of $t_{k}$ thus resulting in the thickening of the shadow of the crack. For coarse or noisy initializations the construction principle presented in Algorithm IS should include an additional substep in Step 4':

- Replace $\rho$ by the updated (sharpened) function $\rho=\rho^{s d}$.

This substep restore the property that $\rho$ is the non-negative distance function of a crack. Its use is successful for slow velocities and short time intervals. However, the disadvantage of this choice lies in the fact that the boundary condition for the solution of the eikonal equation (19) cannot be implemented with high accuracy due to the very slim zero level-set of the shifted function $\rho^{d}$. This has the effect that the resharpening procedure partly neutralizes the propagation step of the WENO scheme. This may lead to a slower propagation than predicted analytically. A larger choice of $d$, on the other hand, gives a faster propagation but a shadow region which is not localized as well as in the first case. The quality of the shadow region is comparable to the result in which we skip the resharpening step 4' completely and put $\rho^{s d}=\rho^{h}$ in (15), and no delay in the speed of propagation is observed.

Note that the function $\rho^{s d}$ obtained with the resharpening process always has the form of a distance function and is, therefore, different in its general form from the function $\rho$ obtained by time-stepping without resharpening. The zero level-sets, however, agree for both.
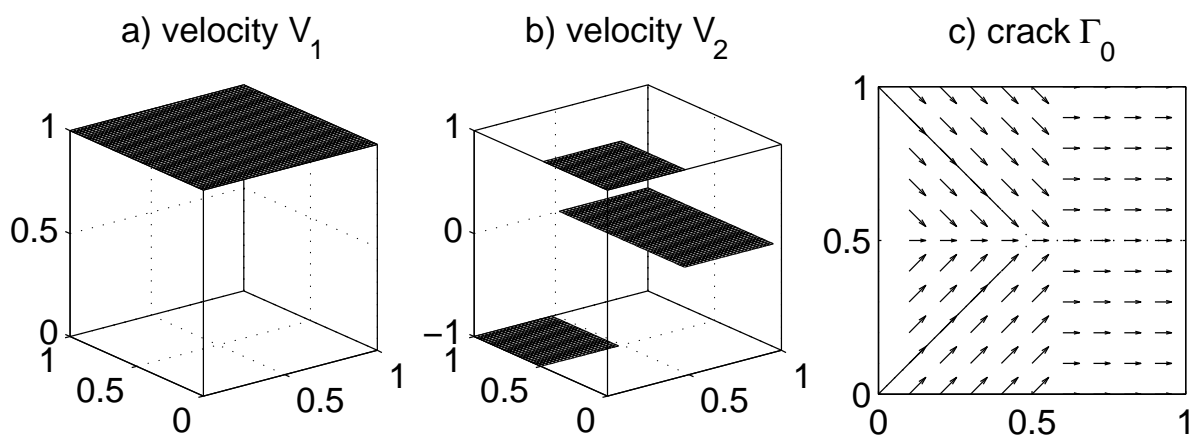

FiguRE 8. Data for the merging crack.

For another non-smooth example we present a merging crack whose corresponding discontinuous velocity is depicted in Fig. 8. The velocity 
is cut off by zero near the boundary $x_{1}=0$. In Fig. 9 we show: a) the initial distance $\left.\rho_{0}, \mathrm{~b}\right)$ the shadow of the initial crack $\Gamma_{0}$ with $\varepsilon=h$, c) distance $\rho, \mathrm{d})$ the shadow of $\Gamma_{t}$ with $\varepsilon=h$, which are computed by Algorithm IS after 500 time-steps for $h=0.01$ and $\Delta t=0.001$. We observe that for this example the resharpening procedure does not have a dominant effect and it can be therefore omitted.
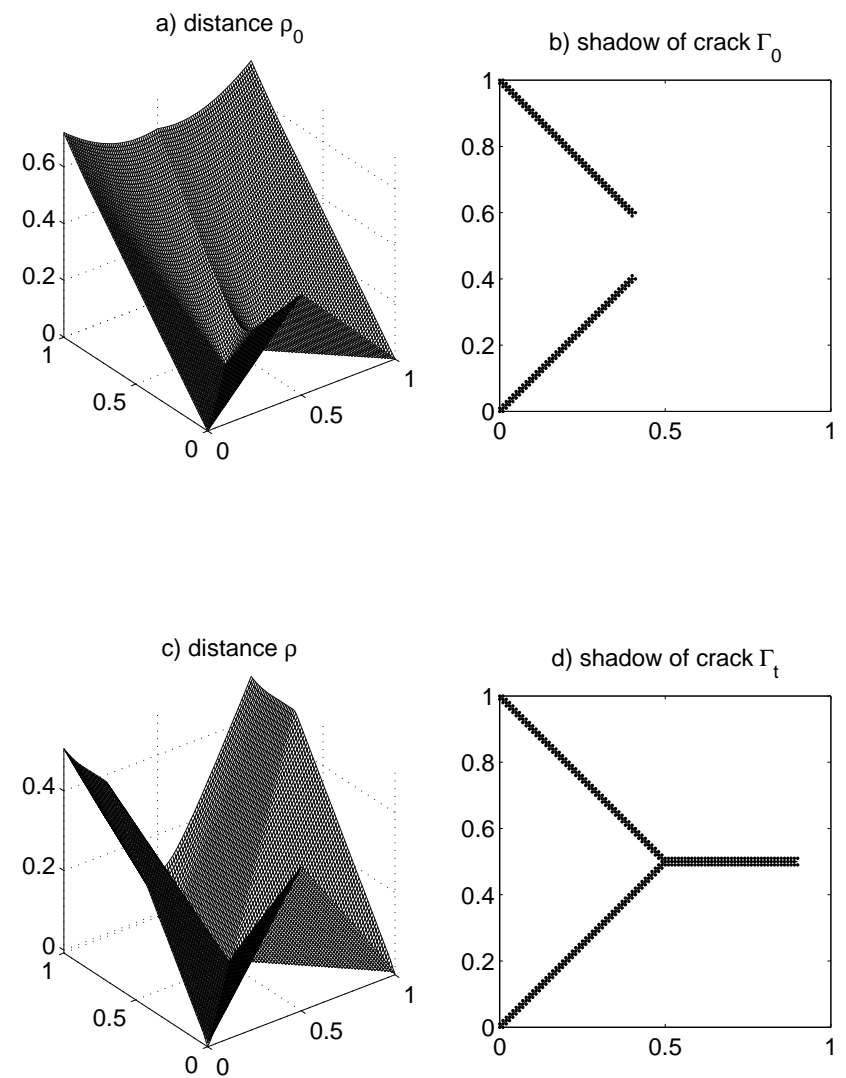

FiguRE 9. The merging crack obtained by Algorithm IS.

Finally, we present a 3D-calculation. A helicoid-shaped crack with in the unite cube $\Omega=\left\{\left(x_{1}, x_{2}, x_{3}\right) \in(0,1)^{3}\right\}$ is considered. First we define parametrically the unbounded surface $\Upsilon_{t}$ for $t \in\left[0,1-t_{0}\right)$ with fixed $0<t_{0}<1$ :

$$
\begin{aligned}
\Upsilon_{t}=\left\{x \in \mathbb{R}^{3}:\left\{\begin{array}{l}
x_{1}=s_{1}, \\
\left(x_{2}-0.5\right) \cos 2 \pi s_{1}-\left(x_{3}-0.5\right) \sin 2 \pi s_{1}=0,
\end{array}\right.\right. \\
\left.\quad \text { for } s_{1} \in\left(-\infty, t_{0}+t\right)\right\}
\end{aligned}
$$


This crack is illustrated in Fig. 10 for $t=1$. Using arguments already presented above we construct the corresponding time-independent velocity

$$
V=\left(1,2 \pi\left(x_{3}-0.5\right),-2 \pi\left(x_{2}-0.5\right)\right)
$$

and cut it off by zero near $x_{1}=0$ to preserve the initial crack. The bounded crack $\Gamma_{t}$ will be defined in a cylinder inside $\Omega$ :

$$
\Gamma_{t}=\Upsilon_{t} \cap\left\{x_{1}>0\right\} \cap\left\{\left(x_{2}-0.5\right)^{2}+\left(x_{3}-0.5\right)^{2}<0.5^{2}\right\} .
$$

For $T=0.9$ we initialize with $t_{0}=0.1$ and parameterize the initial crack $\Gamma_{0}$ by two parameters: $s_{1}$ along the $x_{1}$-axis and $s_{2}$ in the planes $x_{1}=s_{1}$, with uniform spacing $\Delta s$. In $\Omega$ we construct a uniform grid of mesh-size $h$ and discretize the time-interval $[0, T]$ by uniform steplength $\Delta t$. For $h=\Delta t=\Delta s=0.01$ the moving crack $\Gamma_{t}^{h}$ obtained by Algorithm CT after 90 time-steps is depicted in Fig. 10 a). The shadow $\Gamma_{t_{k}}^{h}(\varepsilon)$ for $\varepsilon=\sqrt{2} h$ calculated by Algorithm IS (without resharpening) with $h=0.02, \Delta t=0.002$, and $\Delta s=0.01$ after 450 time-steps is depicted with points in Fig. 10 b). For this 3D-example both algorithms perform successfully.

a) $\operatorname{crack} \Gamma_{t}$

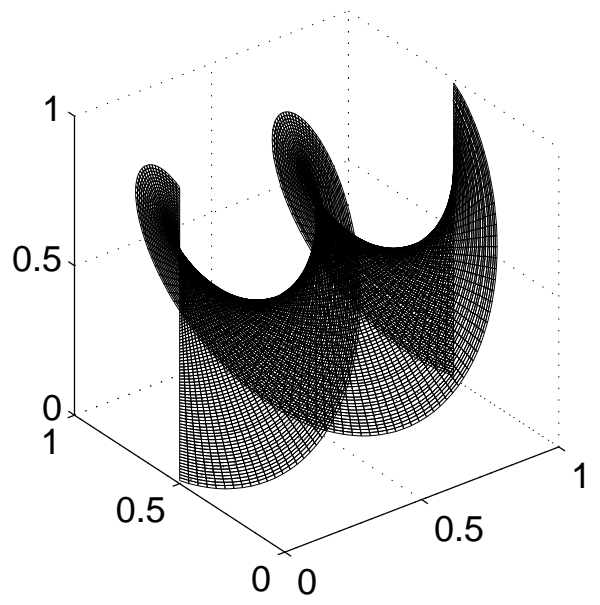

b) shadow of $\operatorname{crack} \Gamma_{t}$

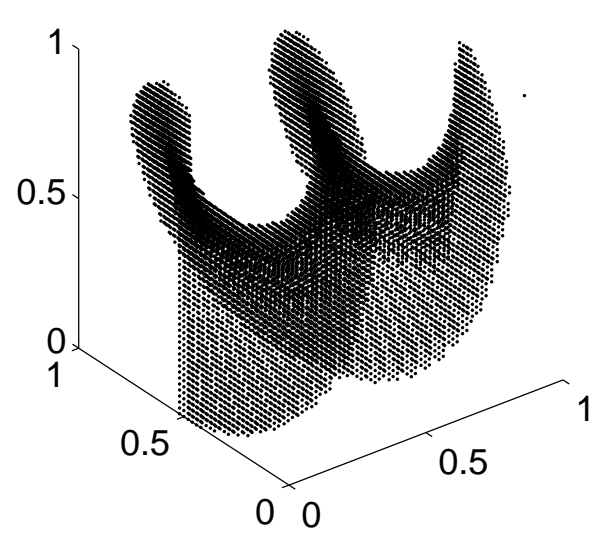

Figure 10. The helicoid-shaped crack obtained by Algorithms CT and IS.

Acknowledgment. The research results were obtained with the support of the Austrian Science Fund (FWF) in the framework of the SFB project F003 "Optimierung und Kontrolle" and the research project 
P18267-N12, and the Russian Foundation for Basic Research (project 06-01-00209).

\section{REFERENCES}

[1] L. Ambrosio and H.M. Soner, Level set approach to mean curvature flow in arbitrary codimension, J. Differential Geom. 43 (1996), 693-737.

[2] L. Ambrosio and H.M. Soner, Flow by mean curvature of surfaces of any codimension, in Variational Methods for Discontinuous Structures, Progr. Nonlinear Differential Equations Appl. 25, 123-134, Birkhäuser, Basel, 1996.

[3] P. Burchard, L.-T. Cheng, B. Merriman and S. Osher, Motion of curves in three spatial dimensions using a level set approach, J. Comput. Phys. 170 (2001), 720-741.

[4] M. Burger, A level set method for inverse problems, Inverse Problems $\mathbf{4 7}$ (2001), 5, 1327-1355.

[5] M. Burger and W. Ring, Foundation of a level-set based shape sensitivity analysis, In preparation.

[6] E.D. Conway, Generalized solutions of linear differential equations with discontinuous coefficients and the uniqueness question for multidimensional quasilinear conservation laws, J. Math. Anal. Appl. 18 (1967), 238-251.

[7] M.G. Crandall and P.L. Lions, On existence and uniqueness of solutions of Hamilton-Jacobi equations, Nonlinear Anal. T M A 10 (1986), 353-370.

[8] M.C. Delfour and J.-P. Zolesio, Shapes and Geometries, SIAM, Philadelphia, 2001.

[9] P.J. DiPerna and P.L. Lions, Ordinary differential equations, transport theory and Sobolev spaces, Invent. Math. 98 (1989), 511-547.

[10] A.F. Filippov, Differential equations with discontinuous right-hand side, Amer. Math. Soc. Transl. Ser.2, Vol. 42, pp.199-231, AMS, Providence, 1964.

[11] A. Friedman and Y. Liu, Propagation of cracks in elastic media, Arch. Rat. Mech. Anal. 136 (1996), 3, 235-290.

[12] M. Hintermüller and W. Ring, A second order shape optimization approach for image segmentation, SIAM J. Appl. Math. 64 (2003/04), 2, 442-467.

[13] K. Ito, K. Kunisch and Z. Li, Level-set function approach to an inverse interface problem, Inverse Problems 17 (2001), 5, 1225-1242.

[14] G.-S. Jiang and D. Peng, Weighted ENO schemes for Hamilton-Jacobi equations, SIAM J. Sci. Comput. 21 (2000), 6, 2126-2143.

[15] A.M. Khludnev and V.A. Kovtunenko, Analysis of Cracks in Solids, WITPress, Southampton, Boston, 2000.

[16] A.M. Khludnev, K. Ohtsuka and J. Sokolowski, On derivative of energy functional for elastic bodies with cracks and unilateral conditions, Quart. Appl. Math. 60 (2002), 1, 99-109.

[17] A.M. Khludnev and J. Sokolowski, Modelling and Control in Solid Mechanics, Birkhäuser, Basel, Boston, Berlin, 1997.

[18] V.A. Kovtunenko, Sensitivity of interfacial cracks to non-linear crack front perturbations, J. Appl. Math. Mech. (ZAMM) 82 (2002), 6, 387-398.

[19] P.L. Lions, Generalized Solutions of Hamilton-Jacobi Equations, Research Notes Math. 69, Pitman, Boston, 1982. 
[20] A. Litman, D. Lesselier and F. Santosa, Reconstruction of a two-dimensional binary obstacle by controlled evolution of a level-set, Inverse Problems $\mathbf{1 4}$ (1998), 685-706.

[21] S. Osher and R. Fedkiw, Level Set Methods and Dynamic Implicit Surfaces, Vol 153 of Applied Mathematical Sciences, Springer, New York, 2003.

[22] S. Osher and J. Sethian, Fronts propagating with curvature dependent speed: Algorithms based on Hamilton-Jacobi formulations, J. Comput. Phys. 79 (1988), 12-49.

[23] S. Osher and C.-W. Shu, High-order essentially nonoscillatory schemes for Hamilton-Jacobi equations, SIAM J. Numer. Anal. 28 (1991), 4, 907-922.

[24] G. Petrova and B. Popov, Linear transport equations with $\mu$-monotone coefficients, J. Math. Anal. Appl. 260 (2001), 307-324.

[25] F. Poupaud and M. Rascle, Measure solutions to the linear multi-dimensional transport equation with non-smooth coefficients, Commun. P.D.Es 22 (1997), 337-358.

[26] J.R. Rice, First-order variation in elastic fields due to variation in location of a planar crack front, J. Appl. Mech. 52 (1985), 571-579.

[27] J.A. Sethian, Level Set Methods: Evolving Interfaces in Geometry, Fluid Mechanics, Computer Vision, and Material Science, Cambridge University Press, 1996.

[28] J.A. Sethian, A fast marching method for monotonically advancing fronts, Proc. Nat. Acad. Sci. 93 (1996), 1591-1595.

[29] J. Sokolowski and J.-P. Zolesio, Introduction to Shape Optimization. Shape Sensitivity Analysis, Springer-Verlag, 1992.

[30] M. Stolarska and D.L. Chopp, Modelling thermal fatigue cracking in integrated circuits by level sets and the extended finite element method, Int. J. Engng. Sci. 41 (2003), 2381-2410.

[31] N. Sukumar, N. Moës, B. Moran and T. Belytschko, Extended finite element method for three-dimensional crack modelling, Int. J. Numer. Meth. Engng. 48 (2000), 1549-1570.

[32] J.N. Tsitsiklis, Efficient algorithms for globally optimal trajectories, IEEE Trans. Automat. Control 40 (1995), 1528-1538.

[33] G. Ventura, J.X. Xu and T. Belytschko, A vector level set method and new discontinuity approximations for crack growth by EFG, Int. J. Numer. Meth. Engng. 54 (2002), 923-944. 